\title{
A PRELIMINARY OPTICAL STUDY OF THE INTERGALACTIC H I CLOUD IN VIRGO
}

\author{
S. DJORGOVSKI I) \\ Palomar Observatory, Division of Physics, Mathematics, and Astronomy, California Institute of Technology, \\ Pasadena, California 91125 \\ Received 21 September 1989
}

\begin{abstract}
A possible optical counterpart of the intergalactic $\mathrm{H}$ I cloud in the Virgo Cluster, discovered by Giovanelli and Haynes, is reported. It is a blue, $17 \mathrm{mag}$, irregular galaxy, and it is located near the peak of the $\mathrm{H}$ I emission. No other optical objects which can be obviously associated with the cloud are found in the area from which the $\mathrm{H} \mathrm{I}$ emission was detected, and no extended, low surface brightness counterpart is seen in these data. If the irregular galaxy is really associated with the $\mathrm{H}$ I cloud, the overall properties of the system would be similar to those of a number of other known gas-rich dwarf galaxies. However, the system has an unusually large hydrogen to light ratio, and a large spatial extent, and it could be dynamically young. Optical spectroscopy is needed in order to establish whether the proposed optical counterpart is indeed correct, and if so, to determine the stage of its chemical evolution.
\end{abstract}

\section{INTRODUCTION}

Giovanelli and Haynes (1989; hereafter referred to as GH) have reported their discovery of a large $\mathrm{H}$ I cloud in the Virgo Cluster, without an obvious optical counterpart. (In this paper the object will be designated V1225 + 018, for the want of a better name.) The cloud dimensions are of a galactic scale, with the neutral hydrogen mass of $\mathscr{M}_{\mathrm{H},} \sim 4 \times 10^{9}$ $\mathscr{M}_{\odot}$, the virial mass estimated from the rotation curve $\mathscr{M}_{\text {tot }} \sim 2.1 \times 10^{10} \mathscr{M}_{\odot}$, and the projected linear size $\sim 200$ $\mathrm{kpc}$, at the assumed distance of $20 \mathrm{Mpc}$. There are no other large galaxies nearby, which may argue against the interpretation of the cloud as a debris of a tidal interaction. Instead, GH advocated the interpretation of the cloud as a protogalaxy, which somehow avoided forming stars up to the present epoch.

If this interpretation is correct, the object would be of a considerable cosmological interest. It would give some support to the currently popular gradual galaxy formation scenarios (e.g., Baron and White 1987; Silk and Szalay 1987; Silk 1987, and references therein; etc.), and present a challenge to many other models. Similar objects could also be responsible for at least some of the damped Ly $\alpha$ absorption systems found at large redshifts (see, e.g., Wolfe 1988, and references therein).

Objects like this must be rare, since no large intergalactic $\mathrm{H}$ I clouds were found so far, in spite of several thorough searches (Lo and Sargent 1979; Sargent and Lo 1985; Hoffman et al. 1989), or even serendipitously in the course of redshift surveys at Arecibo and elsewhere. Schneider and his collaborators (Schneider 1985; Schneider 1989, and references therein) discovered an intergalactic $\mathrm{H}$ I ring in the Leo group, but this object is almost certainly a product of a tidal interaction. Bothun et al. (1987) discovered a gas-rich, low surface brightness disk galaxy, Malin-1, but that object is probably a slowly evolving, rather than a primeval galaxy (Impey and Bothun 1989). Some low-redshift starburst dwarfs, most notably I Zw 18, may be undergoing their first bursts of star formation (Kunth and Sargent 1986, and references therein).

The key observations which could help establish the true nature of V1225 + 018 are likely to be in the optical regime;

a) Alfred P. Sloan Foundation fellow. its immediate distinguishing characteristic is a large hydrogen-to-light ratio. Morphology and luminosity of an optical counterpart, if one is found, can lead and constrain the interpretations of the object. With this in mind, this study was undertaken, using the archival plate material available at Palomar Observatory. The data and the procedures are described in Sec. II.

One dwarf irregular galaxy was found close to the peak of the $\mathrm{H}$ I emission. This object was also found independently by Irwin and McMahon (1989), Bothun (1989), and perhaps also by others. This is not surprising, since the galaxy is clearly visible even on the old Palomar Sky Survey prints. Its optical properties are described in Sec. II. It is proposed here that this galaxy is the principal optical counterpart of $\mathrm{V} 1225+018$. With that assumption made, the properties of $\mathrm{V} 1225+018$ are analyzed and compared to other known objects in Sec. III.

\section{ASTROGRAPHY OF THE REGION}

Only one plate containing the V1225 + 018 position has been taken to date in the course of the Second Palomar Sky Survey. It is a IIIa-F (red) plate; SF01176, taken on UT 1987 April 25. The plate is of a good quality, except for the high fog density, which is irrelevant for this investigation. The region centered on the $\mathrm{H} I$ peak coordinates given by GH was digitized using a PDS scanner at the Jet Propulsion Laboratory. The scan was done in a $4096 \times 4096$ raster mode, with the $16 \mu \mathrm{m}$ square aperture and a $16 \mu \mathrm{m}$ step, giving the pixel scale of 1.074 arcsec. The raster covers completely the $\mathrm{H}$ I cloud as described by $\mathrm{GH}$, and contains seven SAO stars, which can be used for astrometry.

The field also contains the quasar $3 \mathrm{C} 273$, about 34 arcmin from the $\mathrm{H}$ I peak in P. A. $=38^{\circ}$, almost on the extension of the major axis of the cloud. A search for absorption against the quasar may be worthwhile. The position of $3 \mathrm{C} 273$ was used in addition to the SAO stars in computing the coordinate transformations.

The positions of the SAO stars were adjusted for the proper motion estimates, as given in the SAO catalog. Digital centering was performed on the stars, and a bilinear transformation was used to convert the pixel coordinates into the equatorial system, and v. v. Besselian 1950 equinox, 1987.3 epoch coordinates are used throughout. The effective errors 
of the transformations are about 0.9 arcsec in each coordinate. A photoelectric sequence near 3C 273, given by Penston et al. (1971), was used to establish a rough magnitude calibration; in addition, the sky surface brightness can be used to estimate the low-level features; both zero points are uncertain by about $0.5 \mathrm{mag}$ (red).

Background gradients were removed from the scan using median filtering. A variety of simple image-processing techniques was used in order to smooth and display the data, and the whole area corresponding to the cloud outline as given by GH was examined for faint optical features, with a special attention given to the regions of the two $\mathrm{H}$ I maxima, and the cloud center.

No extended emission was found on the large angular scales, between about a half arcmin, and a degree, at a level $\mu_{R} \sim 24 \mathrm{mag} / \mathrm{arcsec}^{2}$, or brighter. This limit is set mainly by the nonuniformities in the emulsion and the plate development. On the scales smaller than a couple of arcminutes, the limiting surface brightness is $\mu_{R} \sim 25 \mathrm{mag} / \mathrm{arcsec}^{2}$. The corresponding blue surface brightness would be about a magnitude fainter. Thus, there is no large, low surface brightness disk associated with V1225 + 018, within the limits of these data. There is also no evidence for a bulgelike condensation near the cloud center, down to $m_{R} \sim 19$, where the confusion by background galaxies becomes important. V1225 + 018 is thus not an analog of Malin-1 (Bothun et al. 1987; Impey and Bothun 1989).
The most interesting optical feature found is an irregular galaxy, denoted here as F-1, practically coinciding with the peak of the $\mathrm{H}$ I emission as found by GH. Its morphology is suggestive of a starburst irregular, and the degree of resolution is consistent with the distance of the Virgo Cluster. The only other optical feature which is probably not a background object is a few arcmin to the $\mathrm{NE}$ from the $\mathrm{H}$ I peak and the galaxy F-1, and is denoted F-2 (cf. Fig. 1). No other believable low surface brightness (LSB) features were found, with one possible exception near the secondary $\mathrm{H} \mathrm{I}$ peak, close to the plate limit, and whose reality is thus somewhat dubious. No interesting objects at all were found near the cloud center.

The region near the $\mathrm{H} \mathrm{I}$ peak is shown in Fig. 1, and a zoom-in on the galaxy F-1 is shown in Fig. 2. The positions and estimated magnitudes of several offset stars and the faint objects are listed in Table I. Positions of additional stars in the region can be provided by the author upon request. The blue magnitudes of the galaxies are probably about $1 \mathrm{mag}$ fainter.

Polaroid charts were made from the old Sky Survey prints for F-1, F-2, and a dozen of Virgo dwarfs of comparable magnitudes, and a range of morphologies, taken from the lists by Reaves (1983), Binggeli, Sandage, and Tarenghi (1984), and Binggeli and Sandage (1984). These comparisons by eye also suggest that the integrated magnitude of F-1 is $m_{B} \sim 17$, or slightly fainter. Its color is very blue, indica-

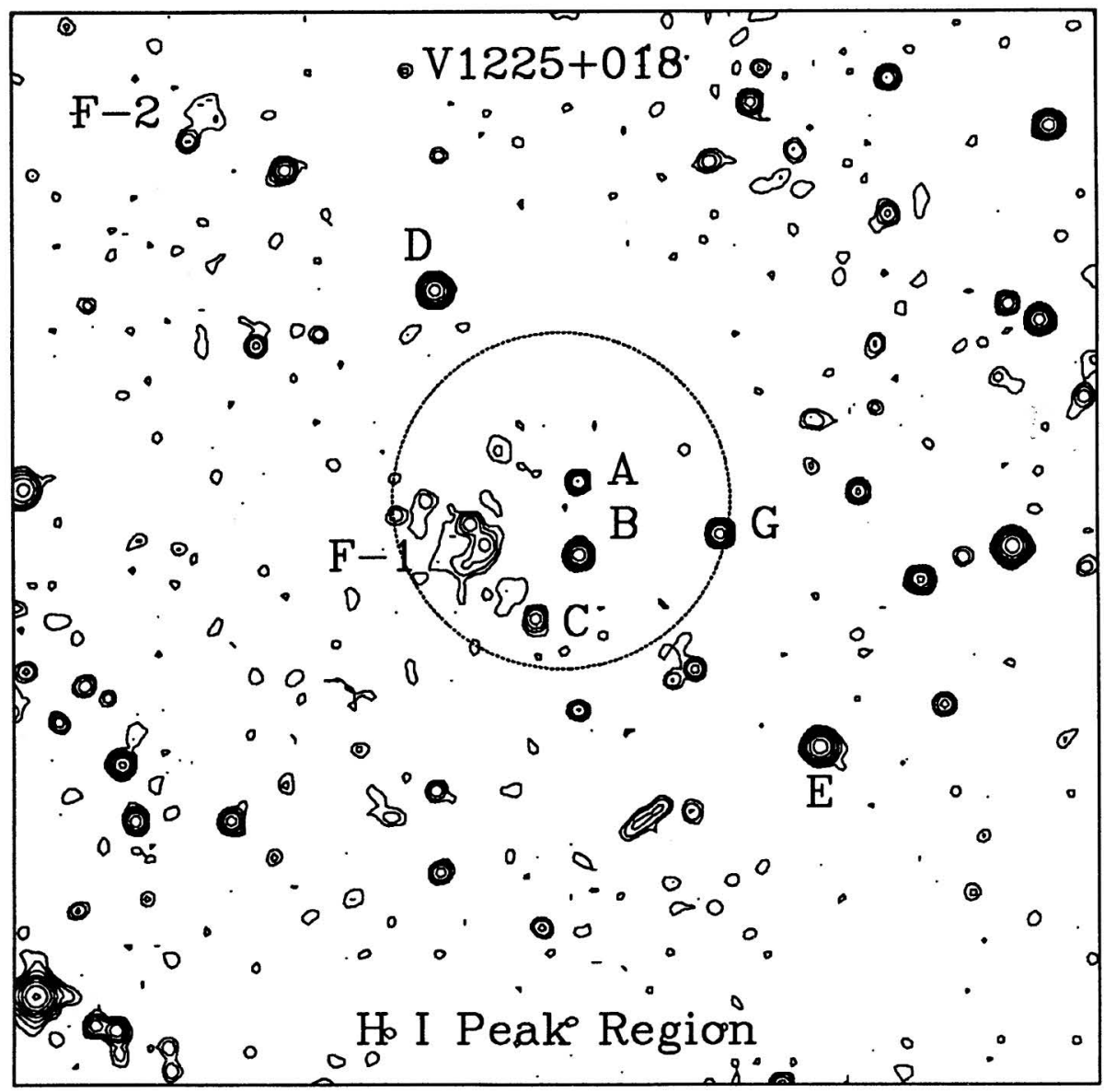

Fig. 1. Section of the plate scan, centered on the $\mathrm{H}$ I peak. The field shown is 550 arcsec square, with $\mathbf{N}$ to the top and $\mathrm{E}$ to the left. The two low surface brightness objects ( $\mathrm{F}-1$ and F-2), and several offset stars are indicated; their coordinates are listed in Table I. The dotted circle corresponds to the Arecibo beam, and is centered on the $\mathrm{H}$ I peak. 


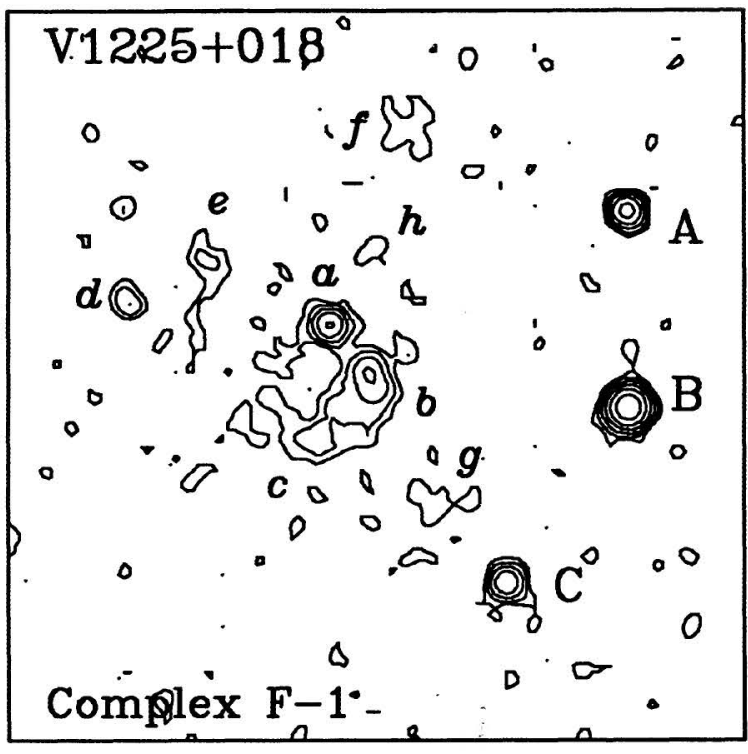

FIG. 2. Zoom-in on the proposed optical counterpart F-1 with its components labeled $(a-h)$; their coordinates are given in Table I. The field shown is 137 arcsec square, with $\mathrm{N}$ to the top and $\mathrm{E}$ to the left. The component $a$ appears stellar (unresolved) in this image. The component $b$ may be a double, and there is another distinct component between $b$ and $c$, but its position could not be measured accurately.

tive of an ongoing star formation. The component F-1 $a$ is very red; it could be a foreground star superposed, or an $\mathrm{H} \alpha$ bright knot, perhaps an $\mathrm{H}$ iI region. None of the objects are detected in any of the IRAS catalogs. Spectroscopy is needed before much more can be said about these objects. CO measurements in the direction of F-1 may also be useful.

What appears to be a background cluster of galaxies is seen to the SSW of the secondary H I peak, near $\alpha=12^{\mathrm{h}}$ $24^{\mathrm{m}} 23^{\mathrm{s}}, \delta=+01^{\circ} 37.0^{\prime}$ (B1950).

TABLE I. Positions for objects near the H I peak.

\begin{tabular}{llllllll}
\hline Object & \multicolumn{2}{c}{$\alpha_{1950}$} & & $\delta_{1950}$ & $m_{R}$ \\
\hline Star A & 12 & 25 & 12.44 & +01 & 52 & 24.2 & 14.8 \\
Star B & 12 & 25 & 09.98 & +01 & 52 & 24.1 & 13.4 \\
Star C & 12 & 25 & 07.82 & +01 & 52 & 46.7 & 14.6 \\
Star D & 12 & 25 & 18.99 & +01 & 53 & 36.6 & 12.6 \\
Star E & 12 & 25 & 03.42 & +01 & 50 & 23.0 & 11.8 \\
Star G & 12 & 25 & 10.67 & +01 & 51 & 12.8 & 14.2 \\
\hline F-1 $a$ & 12 & 25 & 13.18 & +01 & 52 & 47.2 & \\
F-1b & 12 & 25 & 12.49 & +01 & 52 & 40.1 & \\
F-1c & 12 & 25 & 11.81 & +01 & 52 & 49.7 & \\
F-1d & 12 & 25 & 13.47 & +01 & 53 & 24.5 & $16.5:$ \\
F-1e & 12 & 25 & 13.95 & +01 & 53 & 09.8 & \\
F-1f & 12 & 25 & 15.65 & +01 & 52 & 32.4 & \\
F-1 $g$ & 12 & 25 & 10.96 & +01 & 52 & 28.7 & \\
F-1 $h$ & 12 & 25 & 14.09 & +01 & 52 & 39.2 & \\
\hline F-2 star & 12 & 25 & 24.13 & +01 & 55 & 40.6 & $18:$ \\
F-2 fuzz & 12 & 25 & 25.20 & +01 & 55 & 32.0 & \\
\hline \hline
\end{tabular}

\section{DISCUSSION AND COMPARISONS WITH OTHER OBJECTS}

For the purpose of this discussion, it will be assumed that the galaxy F-1 is indeed physically associated with $\mathrm{V} 1225+018$. This is at least plausible, but only the followup spectroscopy can check this association. Since no other obvious optical fragments were found, the total blue magnitude of $17 \pm 1$ will be assumed for the optical counterpart of $\mathrm{V} 1225+018$. The distance scale of $\mathrm{GH}$ will also be adopted $\left(D_{\text {Virgo }}=20 \mathrm{Mpc}\right)$, and $H_{0}=50 \mathrm{~km} \mathrm{~s}^{-1} \mathrm{Mpc}^{-1}$. This implies the absolute blue magnitude $M_{B}=-14.5 \pm 1$ for $\mathrm{V} 1225+018$. The $\mathrm{H}$ I parameters and masses from $\mathrm{GH}$ will be assumed.

Even with this tentative identification, the ratio of the $\mathbf{H}_{\mathbf{I}}$ to the visible light, parametrized as $\left(M_{H} / L_{B}\right) \simeq 16$ in solar units, is rather large. The $\left(M_{\text {tot }} / L_{B}\right)$ is about 85 in solar units, not unlike the mass-to-light ratios measured for some of the Local Group dwarf spheroidals (e.g., Aaronson 1986), or other LSB dwarfs (Sargent and Lo 1985; Kormendy 1987; Carignan and Freeman 1988, etc.)

A comparison in terms of mass-to-light ratios and optical luminosity of V1225+018 with some known spiral and dwarf galaxies is shown in Figs. 3 and 4. The data on normal Virgo spirals are from Giovanelli and Haynes (1983); LSB spirals are from Romanishin et al. (1982); dwarfs from Lo and Sargent (1979) and Sargent and Lo (1985); Malin-1 from Impey and Bothun (1989); DDO 154 from Carignan and Freeman (1988); I Zw 18 from Sargent and Searle (1970) and Lequeux and Viallefond (1980); and DDO 47 and Sextans B from Comte et al. (1985). We note that in some of the dwarfs the velocity fields may be predominantly chaotic, and unknown projection factors enter in the computations, but they can hardly exceed a factor of 2 .

There is a clear trend of increasing $\left(M_{H} / L_{B}\right)$ with decreasing luminosity, which is well known along the Hubble sequence (Fig. 3). The trend continues to the dwarf region, but with an increase in the scatter which is real. $\mathrm{V} 1225+018$ is on the upper envelope of the trend, but otherwise is not exceptional. The trend reflects an increase in star formation efficiency, and therefore the fuel consumption, for the more massive systems, as integrated over the Hubble time. A recent model for the process was presented by Wyse and Silk (1989). In this context, gas-rich dwarfs are galaxies in which star formation was inefficient for the lack of a stimulative dynamical agent, e.g., differential rotation, or a density wave. Of course, evolutionary histories of dwarf galaxies are still quite uncertain, but a general trend would be to move down and to the right on the diagram shown in Fig. 3. This suggests that V1225 +018 may be a relatively young system, but it is not compelling. Many gas-rich dwarfs, V1225 + 018 included, could be regarded as living fossiles of galaxy formation, "puddles of gas" with a delayed star formation (Gallagher and Hunter 1984), but they are not equilvalent to the progenitors of normal, large galaxies of today.

Figure 4 shows a much noisier and shallower trend of dynamical mass-to-light ratios with the luminosity. High $(\mathscr{M} / L)$ ratios in many dwarf galaxies are now reasonably well documented, demonstrating both the presence and the dynamical dominance of dark matter in such systems (Sargent and Lo 1985; Aaronson 1986; Kormendy 1987; Carignan and Freeman 1988). Again, V1225 + 018 is on the upper envelope of the trend, but does not stand out. It is comparable to DDO 145, "a dark galaxy" of Carignan and Freeman (1988). Arguments similar to those above also ap- 


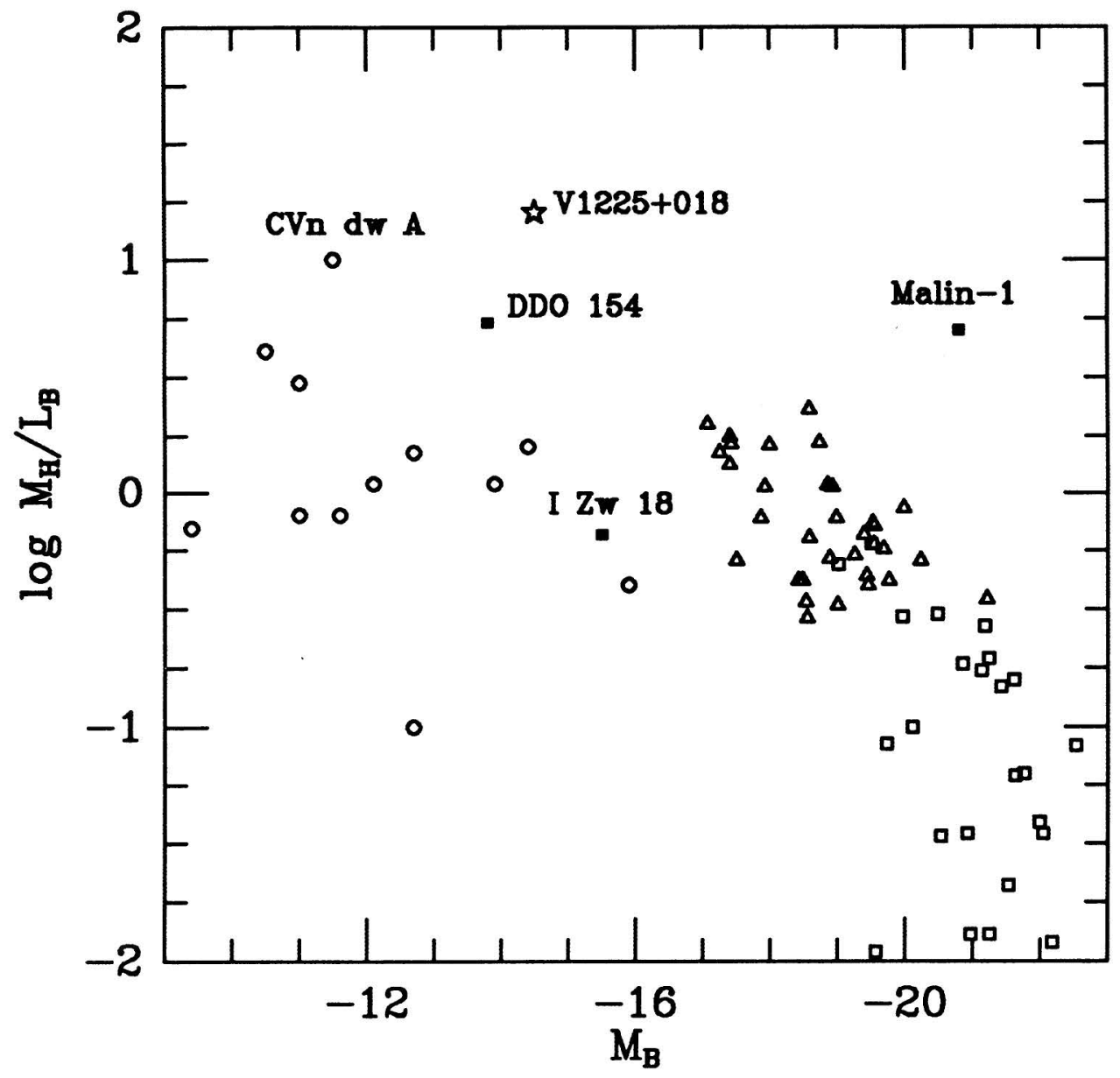

Fig. 3. $\left(\mathscr{M}_{H} / L_{B}\right)$ ratios for different objects plotted against the absolute blue magnitude. Open squares: Virgo spirals from Giovanelli and Haynes (1983). Triangles: LSB spirals from Romanishin et al. (1982). Circles: dwarfs from Sargent and Lo (1985). Other references are given in the text. ply here: dwarfs are relatively inefficient in converting their gas into stars, but extreme $(\mathscr{M} / L)$ ratios do not necessarily imply a young age for any given object, as the Local Group dwarf spheroidals clearly demonstrate.

Finally, we can consider V1225 + 018 and similar objects in the framework of the Tully-Fisher relation (Fig. 5). The same data sources as in Figs. 3 and 4 were used where appropriate, with addition of Virgo spirals from Kenney and Young (1989). The Tully-Fisher relation is a fundamental scaling law between the luminosity and a velocity scale (e.g., the maximum rotational velocity $V_{\max }$ ). The relation represents a connection between the properties of dark halos and the stellar disks, and it reflects both the virial theorem, and the astrophysics of formative processes of disk galaxies. Deviations from a single power law are directly related to the differences in the $(\mathscr{M} / L)$ ratios (e.g., Djorgovski et al. 1988, and references therein). Indeed, the dwarfs deviate from the trend set by the normal spirals, as one might expect from their high $(\mathscr{M} / L)$ ratios. V1225 +018 is well within the locus defined by other gas-rich dwarfs, and does not appear to be exceptional.

The properties of V1225 + 018 are thus similar to those of the known, gas-rich dwarfs, and suggest that it may be a similar kind of an object. This interpretation depends crucially on the assumption that object F-1 is indeed the principal optical counterpart of the $\mathrm{H}$ I cloud discovered by $\mathrm{GH}$.

There is one important distinction, however.
$\mathrm{V} 1225+018$ is much larger in its $\mathrm{H} \mathrm{I}$ extent than any of the known gas-rich dwarfs with comparable optical luminosities (Jaffe et al. 1978; Lequeux and Viallefond 1980; Viallefond and Thuan 1983; Viallefond et al. 1985). Like V1225 + 018, their $\mathrm{H}$ I distributions are often asymmetric and clumpy, but the extent of the gas is only up to a few kpc, 10-100 times less than in $\mathrm{V} 1225+018$. This may suggest that the $\mathrm{H} \mathrm{I}$ is still collapsing in V1225 +018 , and that the object is at least dynamically young. The optical maxima in gas-rich starforming dwarfs are generally coincident with the $\mathrm{H}$ I maxima, except for a small offset, caused perhaps by the photoionization of the $\mathrm{H}$ I by the young stars. Such positional arrangement is also suggested here.

\section{CONCLUSIONS}

A tentative optical counterpart is proposed for $\mathrm{V} 1225+018$, the intergalactic $\mathrm{H}$ I cloud in the Virgo Cluster, discovered by Giovanelli and Haynes (1989). It is a $\sim 17$ mag dIrr galaxy located practically at the maximum of the H I emission. Optical spectroscopy is necessary before this association can be made secure (or otherwise). If the identification is correct, V1225 + 018 would be an extremely gasrich dwarf, an unusual and rare, but not an exceptional object. It still could be a relatively young galaxy, as suggested by its large $\left(\mathscr{M}_{H} / L_{B}\right)$ ratio, and the size of the gas cloud. An important remaining question is whether the system is 

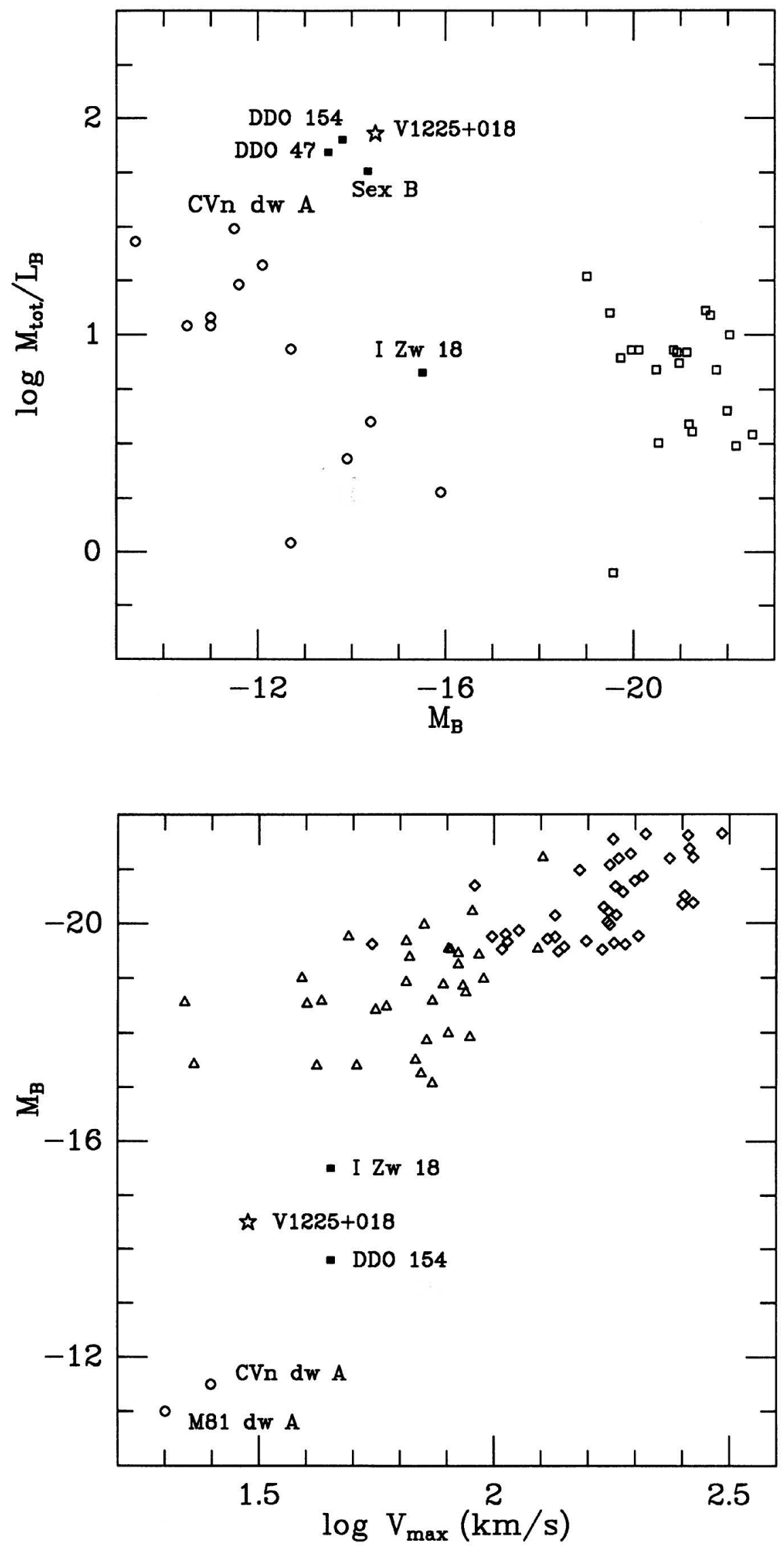

FIG. 4. $\left(M_{\text {tot }} / L_{B}\right)$ ratios for different objects plotted against the absolute blue magnitude. Same symbols as in Fig. 3.
FIG. 5. Tulley-Fisher relation for spiral and dwarf galaxies. Same symbols as in Fig. 3, except for the diamonds, which represent Virgo spirals from Kenney and Young (1989). 
undergoing its first burst of star formation. Abundance measurements from optical spectroscopy could provide an answer to that. In any case, the object is well worth a further study.

This work is based in part on a photographic plate obtained at the Palomar Observatory 48 in. Oschin Telescope for the Second Palomar Observatory Sky Survey, which was funded by the Eastman Kodak Company, the National Geo- graphic Society, the Alfred P. Sloan Foundation, the Samuel Oschin Foundation, the National Science Foundation Grants Nos. AST 84-08225 and AST 87-19465, and the National Aeronautics and Space Administration grants NGL 05002140 and NAGW 1710.

The author would like to thank Joe Fulton of Jet Propulsion Laboratory for scanning the plate, and to Dr. Martha Haynes for providing a preprint of the GH paper. This work was supported in part by the Alfred P. Sloan Foundation and by California Institute of Technology.

\section{REFERENCES}

Aaronson, M. (1986). In Stellar Populations, edited by C. Norman et al. (Cambridge University, Cambridge), p. 45.

Baron, E., and White, S. D. M. (1987). Astrophys. J. 322, 585.

Binggeli, B., and Sandage, A. (1984). Astron. J. 89, 919.

Binggeli, B., Sandage, A., and Tarenghi, M. (1984). Astron. J. 89, 64.

Bothun, G. (1989). Private communication.

Bothun, G., Impey, C., Malin, D., and Mould, J. R. (1987). Astron. J. 94, 23.

Carignan, C., and Freeman, K. (1988). Astrophys. J. Lett. 332, L33.

Comte, G., Lequeux, J., and Viallefond, F. (1985). In Star Forming Dwarf Galaxies and Related Objects, edited by D. Kunth et al. (Editions Frontières, Gif sur Yvette), p. 273.

Gallagher, J., and Hunter, D. (1984). Annu. Rev. Astron. Astrophys. 22, 37.

Giovanelli, R., and Haynes, M. (1983). Astron. J., 88, 881.

Giovanelli, R., and Haynes, M. (1989). Astrophys. J. Lett. (in press) (GH).

Hoffman, G. L., Helou, G., Salpeter, E., and Lewis, B. M. (1989). Astrophys. J. 339, 812.

Impey, C., and Bothun, G. (1989). Astrophys. J. 341, 89.

Irwin, M., and McMahon, R. (1989). As cited by Science News 136, 164. Jaffe, W. J., Perola, G. C., and Tarenghi, M. (1978). Astrophys. J. 224, 808. Kenney, J., and Young, J. (1989). Astrophys. J. 344,171.

Kormendy, J. (1987). In Dark Matter in the Universe, IAU Symposium No. 117, edited by J. Kormendy and G. Knapp (Reidel, Dordrecht), p. 139.
Kunth, D., and Sargent, W. L. W. (1986). Astrophys. J. 300, 496. Lequeux, J., and Viallefond, F. (1980). Astron. Astrophys. 91, 269. Lo, K. Y., and Sargent, W. L. W. (1979). Astrophys. J. 227, 756.

Penston, M. J., Penston, M. V., and Sandage, A. (1971). Publ. Astron. Soc. Pac. 83, 783.

Reaves, G. (1983). Astrophys. J. Suppl. 53, 375.

Romanishin, W., Krumm, N., Salpeter, E., Knapp, G., Strom, K. M., and Strom, S. E. (1982). Astrophys. J. 263, 94.

Sargent, W. L. W., and Lo, K. Y. (1985). In Star Forming Dwarf Galaxies and Related Objects, edited by D. Kunth et al. (Editions Frontières, Gif sur Yvette), p. 253

Sargent, W. L. W., and Searle, L. (1970). Astrophys. J. Lett. 162, L155.

Schneider, S. (1985). Astrophys. J. Lett. 288, L33.

Schneider, S. (1989). Astrophys. J. 343, 94.

Silk, J. (1987). In Observational Cosmology, IAU Symposium No. 124, edited by A. Hewitt et al. (Reidel, Dordrecht), p. 391.

Silk, J., and Szalay, A. (1987). Astrophys. J. Lett. 323, L107.

Viallefond, F., Lequeux, J., and Comte, G. (1985). In Star Forming Dwarf Galaxies and Related Objects, edited D. Kunth et al. (Editions Frontières, Gif sur Yvette), p. 139.

Viallefond, F., and Thuan, T. X. (1983). Astrophys. J. 269, 444.

Wolfe, A. (1988). In QSO Absorption Lines: Probing the Universe, edited C. Blades et al. (Cambridge University, Cambridge), p. 297.

Wyse, R. F. G., and Silk, J. (1989). Astrophys. J. 339, 700. 